\title{
Diferencias evolutivas en procesamiento de textos mediante una tarea de ordenación de frases
}

\author{
EDUARDO VIDAL-ABARCA \\ Universidad de Valencia

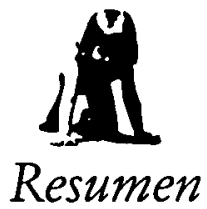

Este estudio presenta los resultados de una investigación en la que se describen algunas diferencias evolutivas en el procesamiento de la prosa expositiva. Treinta y cinco chicos de diferentes edades, 12 de $5 .^{\circ}$ de E.G.B., 12 de $8^{\circ}$ de E.G.B. y 11 de $3^{\circ}$ B.U.P. y F.P. (10, 13 y 16 años respectivamente), realizaron una tarea de ordenación de frases para formar un texto coherente. Habia un total de tres textos de tipo expositivo, constando cada uno de seis oraciones. Se tomaron varias medidas en cada texto: una primera de ordenación global, otra de supraordenación, una tercera de coherencia local (conexiones entre seis pares de frases), y una última medida de errores de conexión. Los resultados confirman una clara tendencia evolutiva en todas las medidas empleadas. Finalmente, se discuten los resultados relacionándolos con otros estudios que analizan el desarrollo de estrategias de comprensión, y se comentan varias repercusiones educativas del trabajo, asi como algunas cuestiones abiertas.

Palabras clave: Procesamiento de textos; Estrategias de comprensión lectora; Desarrollo cognitivo.

\section{Developmental differences in text processing through a sentence arrangement task}

\section{Abstract}

Results from a study showing some developmental differences in processing expository texts as are described. Thirty-five students from different school grades (12 from 5 th, 12 from 8 th, and 11 from 11 th grade) carried out a sentence arrangement task with the object of creating a coherent text. The task consisted in a total of three expository text with six sentences each. For each text several measures were taken: General sentence arrangement, superordination, local coberence (links between six pairs of sentences), and sentence conexion errors. Results confirm a clear developmental trend in all measures. Finally, results are discussed in relation to other researc $b$ work analyzing the development of comprebension strategies. Some educational repercussions and open issues raised in this work are commented.

Keywords: Text processing; Reading comprebension strategies; Cognitive development.

Agradecimientos: Agradezco los comentarios críticos que J. A. García Madruga y J. L. Miralles efectuaron sobre una versión anterior de este trabajo. Dichos comentarios han servido para mejorar la claridad, coherencia y precisión del mismo. Queda a salvo, no obstante, su responsabilidad sobre la versión actual la cual es atribuible exclusivamente al autor.

Correspondencia con autor-es: Universidad de Valencia. E. U. de Formación del Profesorado de E.G.B. C/ Alcalde Reig, 8. 46006 Valencia.

Original recibido: Septiembre 1991. Revisión recibida: Marzo 1992. Aceptado: Mayo 1992. 
De acuerdo con el modelo de procesamiento de textos de van Dijk y Kintsch (1983), los lectores fluidos aplican mecanismos de coherencia local y global durante la lectura. Los primeros implican el establecimiento de relaciones semánticas entre las sucesivas oraciones de un texto a partir de las microproposiciones que se van formando durante la lectura. Los mecanismos de coherencia local son condicionados y a su vez condicionan los mecanismos de coherencia global que producen la macroestructura textual, es decir, una descripción semántica abstracta del contenido global del texto que implica establecer relaciones jerárquicas entre las microproposiciones y las macroproposiciones que las engloban. La construcción de la macroestructura textual supone la existencia de unos procesos abajo-arriba, es decir procedentes de la información textual y otros arribaabajo procedentes del conocimiento de los sujetos. Los primeros se ven facilitados, entre otros elementos, por las señales estructurales, o por la presencia de proposiciones que expresen claramente las macroproposiciones textuales. Los procesos arriba-abajo son posible por los conocimientos del lector, los cuales pueden ser de tipo sintáctico, semántico, episódico o esquemático. El resultado final de ambos mecanismos de coherencia local y global es la elaboración de un modelo situacional entendido como una representación del texto que se integra en el conocimiento del lector. Es decir, coincidiendo en esto con otras explicaciones de la comprensión basados en la construcción de modelos mentales, el lector formaría un escenario mental que incluiría no sólo información textual sino toda aquella que el texto activara en la mente del sujeto (Sanford y Garrod, 1982). Este escenario, modelo situacional o modelo mental se iría construyendo desde los primeros momentos de la lectura y permitiría la realización de inferencias para conectar unas oraciones con otras.

La tarea de ordenación de frases es un medio adecuado para estudiar estos procesos de coherencia local y global por cuanto, en la medida que comprender es esencialmente estructurar y organizar información, las diferentes ordenaciones de frases nos permitirán aproximarnos al modelo mental y a los mecanismos de conexión que están implícitos en la comprensión de los sujetos.

Scardamalia y Bereiter (1984) investigaron la evolución de estos procesos de comprensión con sujetos de $66^{\circ}$ y $10^{\circ}$ grado (12 y 16 años respectivamente) mediante esta tarea de ordenación de frases. Estos autores utilizaron dos textos uno narrativo y otro expositivo con una metodología de pensar-en-voz-alta. En sus conclusiones afirman que chicos de $6 .^{\circ}$ utilizaban procedimientos inmaduros, procesando los textos elemento a elemento, estableciendo conexiones del tipo «tópico-más-detalles» y uniendo frases básicamente por repetición de términos. Es decir, estos lectores parecían no captar el significado global del texto, llegando únicamente a captar «aquello de lo que el texto trata». Por el contrario, los alumnos de $10 .^{\circ}$ grado podían ser calificados en mayor medida como lectores maduros. Estos chicos establecían conexiones entre frases en base a una coherencia global del discurso que se manifestaba por expresiones de resumen que abarcaban varias frases, agrupaban oraciones antes de la colocación definitiva, y verbalizaban inferencias en la justificación de la colocación de las frases. Así, se podía decir que estos lectores captaban «lo que el texto dice acerca del tópicon, es decir, construían las macroproposiciones estableciendo una jerarquía de importancia entre las oraciones a partir del significado global.

Esta investigación plantea, a nuestro modo de ver, algunos problemas que queremos resaltar ya que son los que justifican nuestro estudio. En primer lugar, aunque Scardamalia y Bereiter analizan algunos casos de sujetos que se en- 
cuentran en el estadio de transición entre la utilización de estrategias de procesamiento inmaduras y la aplicación de otras maduras, la diferencia de edad entre los dos grupos de sujetos (12 y 16 años) es, posiblemente, demasiado grande para estudiar dichos procesos de transición. Los chicos que están en esta fase de transición se caracterizarían, siguiendo a estos autores, por el empleo de algunas estrategias maduras pero carecerían de los procedimientos ejecutivos de alto nivel que les podrían permitir producir una representación coherente del texto. El estudio de estos procesos de transición requerían, según nuestro criterio, variar los grupos de edad manteniendo el de sujetos mayores (16 años) pero incluyendo sujetos menores y mayores de 12 años con el fin de tener una perspectiva más clara sobre la evolución de la estrategias de comprensión.

Otro problema del estudio que estamos comentando se refiere al tipo de textos utilizado. Así, Scardamalia y Bereiter emplean, como ya dijimos, un texto narrativo y otro expositivo. Estos autores afirman que, en general, los procesos de ordenación son idénticos para ambos textos aunque ellos mismos encuentran algunas diferencias, como por ejemplo un mayor número de expresiones de resumen en la ordenación del texto expositivo que en la del texto narrativo. La distinción entre ambos tipos de prosa, narrativa y expositiva, ha sido ampliamente reseñada (Brewer, 1980; Mayer, 1985). Esta distinción se apoya en diversas características diferenciales que pueden afectar a la comprensión, y especialmente a la conexión entre frases, tales como el empleo de referentes espaciales y temporales, la cantidad y calidad de las inferencias, o las señales estructurales, entre otras (Graesser y Goodman, 1985; Spiro y Taylor, 1987). Así, mientras las narraciones tienen características retóricas más fijas y ligadas a la descripción de hechos en secuencia temporal, la prosa expositiva presenta unos contenidos informativos mediante una organización lógica o cuasi lógica con una estructura muy variada. Así, siguiendo a Meyer (1985), podríamos distinguir diversas estructuras: compilación (incluyendo enumeración y secuencia), causación, comparación/contraste, descripción y problema/solución. Mencionamos estas estructuras porque, aunque no hay un acuerdo general, hay autores que afirman que algunas de ellas son comprendidas más tempranamente que otras (Englert y Hiebert, 1984; Englert y Thomas, 1987; Richgels y col., 1987). Sería conveniente, por tanto, incluir textos expositivos con diferente estructura para analizar si este hecho altera los resultados en la tarea de ordenación de frases.

En tercer lugar, como antes dijimos, Scardamalia y Bereiter emplean casi exclusivamente medidas metacognitivas basadas en un análisis de protocolos obtenidos con la metodología de pensar-en-voz-alta mientras los chicos realizaban la tarea de ordenación. Esta metodología supone un avance respecto a la entrevista metacognitiva pero no está exenta de problemas (ver Garner, 1987). De hecho, Garner y Gillingham (1987) realizaron una investigación con una tarea muy similar a la de Scardamalia y Bereiter que aporta datos de interés. Estos autores presentaron a niños de $5 .^{\circ}$ y $7 .^{\circ}$ grado (11 y 13 años respectivamente) dos grupos de 7 frases cada uno, un grupo por cada texto, siendo ambos de tipo expositivo. Cinco de las frases formaban parte del texto, siendo una oración la idea principal y estando relacionadas las otras cuatro frases, dos a dos, por referentes anafóricos. Las dos oraciones restantes eran inadecuadas. A los niños se les pedía formar un buen texto y otro malo, pudiendo excluir oraciones. Tomaron medidas de relación temática según los chicos excluyeran o no las oraciones inadecuadas, de supraordenación o colocación de la idea principal al comienzo del texto, y de cohesión, si colocaban las frases unidas por referentes anafóricos 
en el orden debido. Así mismo, tomaron medidas metacognitivas basadas en informes verbales. Los autores encontraron diferencias entre los cursos en las medidas de relación temática y en el informe verbal de la supraordenación. Además, únicamente el $50 \%$ de los niños lograron puntuaciones aceptables de rela. ción temática y supraordenación, bajando esta cifra al $17 \%$ en las puntuaciones de cohesión. El dato que más nos interesa resaltar aquí es que se encontró poca relación entre las puntuaciones de ejecución y las de informe verbal. Estas conclusiones confirman la necesidad de incluir medidas de ejecución junto a medidas metacognitivas, al menos en el caso de sujetos de grados escolares medios.

Así pues, nuestro estudio pretende, en parte, replicar el efectuado por Scardamalia y Bereiter. Al igual que éste, se trata de una investigación descriptiva pero introduce algunas modificaciones congruentes con los problemas que acabamos de señalar. En primer lugar, se incluyen tres niveles de edad (10, 13 y 16 años) con el fin de intentar captar con más detalle los procesos de transición en la aplicación de estrategias de comprensión. En segundo lugar, se emplean sólo textos expositivos, los cuales tienen unas características diferentes de los narrativos, y son los propios de las tareas de aprendizaje escolar. En tercer lugar, utilizamos textos con diferente organización para comprobar si este hecho produce efectos diferenciales en los resultados. Por último, incluimos medidas de ejecución junto a otras metacognitivas si bien aquí nos centramos en el análisis de las primeras por las causas que comentaremos al hablar del procedimiento seguido en la metodología.

Las hipótesis de partida están en la misma línea que las conclusiones de los estudios citados aunque tienen en cuenta las particularidades que acabamos de señalar. En primer lugar, la ordenación de tarjetas para formar un texto coherente debería tener una dimensión evolutiva entre los tres niveles de edad escogidos. En segundo lugar, la medida de supraordenación, la cual sería un indicador de un inicio de estrategias maduras de procesamiento, debería mostrar diferencias evolutivas, sobre todo entre el primer nivel de edad y los dos restantes. En tercer lugar, los procedimientos de coherencia local deberían marcar diferencias evolutivas dependiendo del grado de explicitación en la conexión entre frases. Finalmente, no teníamos una hipótesis previa acerca de los resultados que se obtendrían con los diferentes tipos de textos empleados.

\section{METODOLOGIA}

\section{Sujetos}

Participaron en el estudio un total de 35 sujetos, 12 de $5 .^{\circ}$ de E.G.B., 12 de $8 .^{\circ}$ de E.G.B. y 11 de $3 .^{\circ}$ de B.U.P. ó F.P. ( 6 del primer curso y 5 del otro) de 10,13 y 16 años respectivamente, siendo escogidos aleatoriamente de entre aquellos que tenían un nivel académico medio. La prueba fue aplicada durante el mes de Diciembre del año 1990.

\section{Materiales}

Se seleccionaron seis textos breves de libros de Ciencias Naturales de los cursos medios de E.G.B. y de una enciclopedia infantil. Todos ellos fueron adaptados para procurar que hubiera una única ordenación correcta, y que pudieran descomponerse en seis oraciones. Dichos textos fueron aplicados a una muestra 
de alumnos de la E.U. del Profesorado de E.G.B. con el fin de proceder a una selección de los más adecuados en virtud de la coincidencia en la ordenación. Finalmente seleccionamos tres en los que había un mayor grado de coincidencia en la ordenación, en torno a un $70 \%$ de media. Estos mismos tres textos fueron examinados independientemente por tres profesores del area de filología de la Escuela Universitaria, los cuales coincidieron en la ordenación correcta pensada inicialmente. Los textos se pueden ver en la Figura 1.

\section{FIGURA 1}

\section{(TEXTO 1: ECOSISTEMAS)}

(1) Es muy importante que los ecosistemas estén en equilibrio, es decir, que todas las especies animales y vegetales que viven en una zona tengan posibilidades de sobrevivir.

(2) Para conservar el equilibrio de los ecosistemas el hombre puede tomar dos tipos de medidas: evitar su destrucción y corregir los desequilibrios naturales.

(3) Para evitar la destrucción de los ecosistemas hay que procurar que no mueran los seres vivos que viven en él.

(4) Así, por ejemplo, se puede impedir que se corten árboles, se puede prohibir la caza de especies en peligro de desaparición o tomar medidas para diminuir la contaminación.

(5) La otra medida consiste en corregir los desequilibrios naturales.

(6) Para ello se sueltan crias de peces en los rios y lagos con el fin de repoblarlos o se construyen muros para evitar la erosión.

(TEXTO 2: HURACAN)

(1) Un huracán es una furiosa tempestad de viento que comienza en el mar, pero a diferencia del tornado, el huracán abarca mucho espacio.

(2) Suele extenderse a través de 480 a 650 kilómetros a la vez.

(3) Los furiosos vientos del huracan pueden provocar la formación de grandes olas en el océano.

(4) El viento y las olas pueden hundir los barcos.

(5) Los huracanes también suelen ocasionar grandes lluvias.

(6) Estas lluvias, lo mismo que las olas altas, pueden causar grandes inundaciones.

(TEXTO 3: SONIDO)

(1) El sonido es producido por la vibración de los cuerpos.

(2) Si haces sonar una campana de cristal e inmediatamente acercas débilmente la uña al borde de la copa, sentirás la vibración.

(3) Esa vibración se traslada a través del aire, del agua o de un sólido y llega a nuestro oído.

(4) Excita el tímpano, haciendo que éste vibre también.

(5) La vibración del tímpano pasa a la cadena de huesecillos del oído y de éstos al nervio auditivo.

(6) El nervio transmite esta sensación a nuestro cerebro y así podemos oír.

Textos empleados en la tarea de ordenación de frases

Como se puede ver, el texto «Ecosistemas» es de tipo problema/solución con un problema central, «la necesidad de conservar el equilibrio de los ecosistemas» y una eriumeración de soluciones. El texto "Huracanes» es de tipo causa/efecto, definiendo en primer lugar algunas de sus características y explicando a continuación algunos de sus efectos. El texto «Sonido» es de tipo secuencial, explicando cómo se produce el sonido a partir de la vibración que producen los cuerpos, hasta la llegada de la vibración al oido, los procesos en el interior 
del oido hasta el nervio auditivo, y finalmente la transmisión de la sensación al cerebro.

Cada una de las frases estaba escrita en una tarjeta distinta con letra ordinaria de 14 puntos. Así mismo, había cinco tarjetas iniciales que representaban diversas secciones del dibujo de un «tren». Estas tarjetas se utilizaron para entrenar a los chicos en la tarea de ordenación.

\section{Procedimiento}

Tras explicar a los muchachos la tarea que habían de realizar, se les presentaban las cinco tarjetas iniciales del dibujo del tren a modo de entrenamiento. A continuación se les entregaban las tarjetas correspondientes a cada uno de los textos ordenadas de forma aleatoria, pero con una ordenación idéntica en cada uno de los textos. Se pidió a los chicos que fueran verbalizando tanto la ordenación de las tarjetas, las cuales tenían un color de identificación, así como todo lo que fueran pensando para resolver la tarea. Se balanceó la presentación de los diferentes textos de forma que, en cada grupo, cada uno de los textos fuera leido 4 veces en posición inicial, 4 en segundo lugar y otras cuatro en tercero, con una distribución de 4-4-3 en el caso de los chicos mayores, ya que, como se recordará, había únicamente 11 sujetos. Los examinadores fueron estudiantes de la E.U. de Formación del Profesorado previamente entrenados. Se instruyó a los examinadores para que animaran a los chicos a pensar en voz alta y, en caso de que no lo hicieran así, para que preguntaran ellos directamente acerca de las razones de los muchachos sobre la colocación de las tarjetas. Todo el proceso de aplicación fue grabado magnetofónicamente para poder ser analizado posteriormente. Dado que algunos chicos apenas verbalizaron sus pensamientos y que algunos examinadores o bien no preguntaron en el momento oportuno o bien no aclararon suficientemente las respuestas de los muchachos, se ha optado por presentar únicamente los datos de ejecución.

\section{Medidas y sistema de puntuación}

Se tomaron cuatro tipos de medidas: ordenación del conjunto del texto, supraordenación o colocación de la tarjeta inicial, conexiones locales entre tarjetas y errores de ordenación.

a) Ordenación del texto. Esta medida consistía en una puntuación ordinal de las diferentes ordenaciones que los chicos hacían con cada uno de los textos. El rango de puntuación ordinal iba desde 0 (ordenación muy incorrecta) hasta 4 (ordenación totalmente correcta). Los criterios para valorar la calidad de la ordenación eran dos: conservar el orden de la primera o primeras tarjetas, dependiendo del texto, en la posición correcta, y conservar las conexiones que se consideraron fundamentales para la comprensión del texto, o bien evitar algunos errores de conexión que perjudicaban gravemente la coherencia global. En el Anexo se pueden consultar los criterios de puntuación empleados.

b) Supraordenación. Se otorgaba 1 punto si la primera tarjeta estaba en posición inicial, y 0 en caso contrario.

c) Conexiones locales. Aquí únicamente hemos considerado seis conexiones locales en las cuales existe una relación anafórica, con un grado mayor o menor de explicitación. Pensamos que este análisis nos podía dar pistas acerca de los mecanismos de coherencia local y su evolución. Estas conexiones locales perte- 
necen en su mayoría al texto «Sonido» dada su estructura secuencial, siendo las siguientes: tarjetas 1-3 ó 2-3, 3-4, 4-5 y 5-6. Del texto «Huracanes» contabilizamos las conexiones $1-2$ y $5-6$. Se daba 1 punto si estaba presente y 0 en caso contrario.

d) Errores de ordenación. Analizamos algunos errores que pueden ser particularmente significativos por cuanto pueden informar sobre los mecanismos de coherencia empleados o bien sobre el dominio de claves lingüísticas generales. Se revisa la conexión 2.5 o 5.2 del texto «Ecosistemas», y la colocación de las tarjetas 4 ó 5 antes de la 3 en el texto «Sonido», aunque no fuera inmediatamente antes (p.e. 1-5-4-6-2-3). Así mismo, nos ha parecido interesante contabilizar los niños que colocaron como tarjeta inicial alguna oración que es claramente inadecuada porque hace referencia a una información anterior. Ejemplos de ello sería colocar como tarjeta incial de «Ecosistemas» las tarjetas 4, 5 ó 6, o bien las tarjetas 2, 5 ó 6 de Huracanes, o bien las tarjetas 3-4-5 ó 6 de «Sonido». En todos los casos se puntuaba 1 si estaba presente el error y 0 en caso contrario.

\section{RESULTADOS}

Ordenación del texto. Los resultados de la puntuación de ordenación se pueden ver en la Tabla I. Como se puede apreciar, existe una progresión creciente de una ordenación más correcta conforme avanza el nivel académico. Hay que notar, sin embargo que no hay ningún sujeto de $8 .^{\circ}$ que ordene correctamente las tarjetas del texto «Huracanes». Ello puede deberse a la evolución del conocimiento sintáctico y su papel en esta tarea, tal como comentaremos en la discusión de los resultados, relacionando este dato con otros.

TABLA I

Frecuencia de sujetos en la medida de ordenación de frases $\left(N_{5.0}=12, N_{8.0}=12, N_{3.0^{\circ}}=11\right)$

\begin{tabular}{|c|c|c|c|c|c|c|c|c|c|c|c|c|c|c|c|}
\hline \multirow[b]{2}{*}{ Ordenación } & \multicolumn{5}{|c|}{ Texto «Ecosistemas» } & \multicolumn{5}{|c|}{ Texto «Huracanes» } & \multicolumn{5}{|c|}{ Texto «Sonido» } \\
\hline & 0 & 1 & 2 & 3 & 4 & $\mathbf{0}$ & 1 & 2 & 3 & 4 & $\mathbf{0}$ & 1 & 2 & 3 & 4 \\
\hline 5. E.G.B. & 9 & 1 & 1 & 1 & 0 & 4 & 6 & 1 & 1 & 0 & 7 & 2 & 1 & 2 & 0 \\
\hline 8..$^{\circ}$ E.G.B. & 2 & 4 & 2 & 1 & 3 & 2 & 5 & 1 & 4 & 0 & 1 & 3 & 3 & 2 & 3 \\
\hline 3. B.U.P. y F.P. & 2 & 2 & 2 & 3 & 2 & 0 & 2 & 1 & 4 & 4 & 0 & 2 & 2 & 3 & 4 \\
\hline
\end{tabular}

Dado el bajo número de sujetos en muchas casillas, lo cual hacía inadecuado aplicar una prueba de chi-cuadrado para ver las diferencias entre grupos, agrupamos los sujetos según superaran o no la mediana en cada texto (Siegel, 1978), puntuando con 1 en el primer caso y con 0 en el segundo. Hay que advertir que en caso del texto «Sonido» las puntuaciones se agruparon con el criterio de «sujetos que no alcanzaban la mediana» (0) y «sujetos que llegaban y superaban la mediana» (1) dado que de esta forma se conseguía la división más aproximada en dos mitades. Estos datos se pueden ver en la Tabla II donde figuran las frecuencias absolutas y los porcentajes correspondientes. El índice chi-cuadrado (g.l. 2) con éste último tipo de agrupación fue de 5.6 (n.s.), 9.9, $\mathrm{p}<.01$ y $7.4, \mathrm{p}<.05$ para los textos «Ecosistemas», «Huracanes» y «Sonido» respectivamente. Si comparamos las frecuencias de sujetos en cada par de cursos mediante la prueba de la probabilidad exacta de Fisher obtenemos diferen- 
cias significativas entre los chicos de $50^{\circ}$ de E.G.B. y $30^{\circ}$ de B.U.P.F.P. en todos los textos $(\mathrm{p}<.05, \mathrm{p}<.01$ y $\mathrm{p}<.01$ para «Ecosistemas», «Huracanes» $\mathrm{y}$ «Sonido» respectivamente), diferencias entre $5 .^{\circ}$ y $8 .^{\circ}$ de E.G.B. en «Sonido» $(\mathrm{p}<.05)$, y entre $8 .^{\circ}$ de E.G.B. y $3 .^{\circ}$ de B.U.P.F.P. en «Huracanes» $(p<.05)$

\section{TABLA II}

Frecuencia y porcentaje de sujetos en la medida de ordenación de frases con los datos agrupados según superaran la mediana (1) o no la superaran $(0)\left(N_{5.0}=12, N_{8.0}=12, N_{3.0}=11\right)$

\begin{tabular}{|c|c|c|c|c|c|c|}
\hline \multirow[b]{2}{*}{ Puntuación } & \multicolumn{2}{|c|}{ Texto «Ecosistemas } & \multicolumn{2}{|c|}{ Texto «Huracanes» } & \multicolumn{2}{|c|}{ Texto «Sonido» } \\
\hline & 0 & 1 & 0 & 1 & 0 & 1 \\
\hline $5^{\circ}$ E.G.B. & $\begin{array}{l}10 \\
83\end{array}$ & $\begin{array}{r}2 \\
17\end{array}$ & $\begin{array}{l}10 \\
83\end{array}$ & $\begin{array}{r}2 \\
17\end{array}$ & $\begin{array}{r}9 \\
75\end{array}$ & $\begin{array}{r}3 \\
25\end{array}$ \\
\hline $8^{\circ}$ E.G.B. & $\begin{array}{r}6 \\
50\end{array}$ & $\begin{array}{r}6 \\
50\end{array}$ & $\begin{array}{r}7 \\
58\end{array}$ & $\begin{array}{r}5 \\
42\end{array}$ & $\begin{array}{r}4 \\
33\end{array}$ & $\begin{array}{r}8 \\
67\end{array}$ \\
\hline $3^{\circ}$ B.U.P.F.P. & $\begin{array}{c}4 \\
36\end{array}$ & $\begin{array}{r}7 \\
64\end{array}$ & $\begin{array}{r}2 \\
18\end{array}$ & $\begin{array}{r}9 \\
82\end{array}$ & $\begin{array}{r}2 \\
18\end{array}$ & $\begin{array}{r}9 \\
82\end{array}$ \\
\hline
\end{tabular}

Md «Ecosistemas»: 1; Md «Huracanes»: 1; Md «Sonido»: 2

Así, se aprecian unas claras diferencias evolutivas en nuestra muestra de sujetos que indican que los chicos mayores ordenan las frases de los textos de forma más adecuada que los de cursos inferiores. Así mismo, si hallamos el coeficiente de correlación de Spearman entre las puntuaciones de ordenación de los tres textos hallamos que los mismos son significativos en todos los casos, resultando unos coeficientes de $0.60(\mathrm{p}<.001)$ entre «Ecosistemas» y «Huracanes», $0.40(\mathrm{p}<.01)$ entre «Ecosistemas» y «Sonido», y $0.50(\mathrm{p}<.002)$ entre «Huracanes» $\mathrm{y}$ «Sonido». Es decir, todo parece apuntar a que estamos midiendo un mismo tipo de procesamiento que es independiente de los textos utilizados, si bien, tal como se puede apreciar en la Figura 2 existen unas diferencias en las tendencias evolutivas que dependen del texto utilizado. Así, mientras que el paso de $5 .^{\circ}$ a $8 .^{\circ}$ de E.G.B. es muy pronunciado en «Ecosistemas» y «Sonido», los cambios entre los tres niveles educativos son más equilibrados en el texto «Huracanes». Comentaremos más ampliamente este dato junto con otros de las restantes medidas en el apartado de conclusiones.

Supraordenación. Como se recordará, con esta medida puntuábamos si los chicos habían colocado la primera tarjeta en posición inicial (1) o no (0). Los datos de la Tabla 3 muestran la frecuencia y porcentaje de sujetos con arreglo a esta colocación. El índice chi-cuadrado (g.l. 2) en los diversos textos es 9.6 $(\mathrm{p}<.01)$ para «Ecosistemas», 5.7 (n.s.) para «Huracanes» y $7.4(\mathrm{p}<.05)$ en «Sonido» 1 . Si comparamos las frecuencias en cada par de cursos. mediante la prueba de Fisher, también aquí obtenemos diferencias significativas entre $50^{\circ}$ de E.G.B. y $3 .^{\circ}$ de B.U.P.F.P. en todos los textos $(\mathrm{p}<.05$, $\mathrm{p}<.05$ y $\mathrm{p}<.01$ para «Ecosistemas», «Huracanes» y .«Sonido» respectivamente), y solamente diferencias entre $5 .^{\circ}$ y $8 .^{\circ}$ de E.G.B. en «Ecosistemas» $(\mathrm{p}<.01)$. Así, pues, de nuevo se encuentran diferencias evolutivas significativas en todos los casos, siendo el texto «Huracanes» el que menos diferencias produce. Es decir, las diferencias entre los cursos dependen del texto, siendo en el caso de «Ecosistemas» muy pronunciadas entre $5 .^{\circ}$ curso y el resto de los niveles, habiendo diferencias más 
$\%$ de sujetos

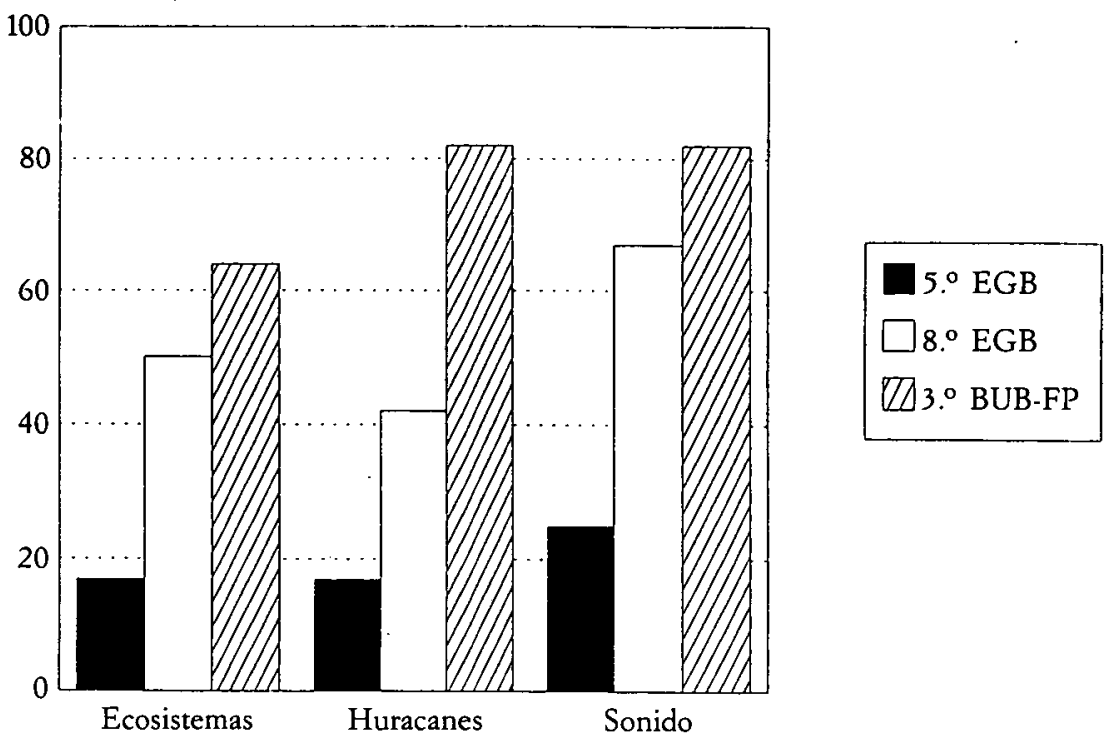

Porcentaje de sujetos de cada curso que superan la mediana de lamedida de ordenación de textos en cada una de los pasajes

\section{TABLA III}

Frecuencia y porcentaje de sujetos en la medida de «supraordenación» $\left(N_{5 . \circ}=12, N_{8.0}=12, N_{3 .}=11\right)$

\begin{tabular}{|c|c|c|c|c|c|c|}
\hline \multirow[b]{2}{*}{ Puntuación } & \multicolumn{2}{|c|}{ Texto «Ecosistemas» } & \multicolumn{2}{|c|}{ Texto «Huracanes» } & \multicolumn{2}{|c|}{ Texto «Sonido» } \\
\hline & 0 & 1 & o & 1 & 0 & 1 \\
\hline $5^{\circ}$ E.G.B. & $\begin{array}{r}9 \\
75\end{array}$ & $\begin{array}{c}3 \\
25\end{array}$ & $\begin{array}{r}5 \\
42\end{array}$ & $\begin{array}{l}7 \\
58\end{array}$ & $\begin{array}{r}9 \\
75\end{array}$ & $\begin{array}{l}3 \\
25\end{array}$ \\
\hline $8 .^{\circ}$ E.G.B. & $\begin{array}{c}2 \\
17\end{array}$ & $\begin{array}{l}10 \\
83\end{array}$ & $\begin{array}{r}3 \\
25\end{array}$ & $\begin{array}{l}9 \\
75\end{array}$ & $\begin{array}{r}6 \\
50\end{array}$ & $\begin{array}{l}6 \\
50\end{array}$ \\
\hline 3. ${ }^{\circ}$ B.U.P.-F.P. & $\begin{array}{r}3 \\
28\end{array}$ & $\begin{array}{l}8 \\
72\end{array}$ & $\begin{array}{l}0 \\
0\end{array}$ & $\begin{array}{c}11 \\
100\end{array}$ & $\begin{array}{r}2 \\
18\end{array}$ & $\begin{array}{l}9 \\
82\end{array}$ \\
\hline
\end{tabular}

escalonadas entre los cursos en los otros dos textos, todo lo cual comentaremos en el apartado de conclusiones.

Conexiones locales. Como ya dijimos, en esta medida contabilizamos si ocurrían (1) o no (0) una serie de conexiones en las que existía una relación anafórica más o menos explícita entre frases. Hay tres conexiones menos explícitas (HUR 1-2, SON 3-4 y SON 4-5) ya que la conexión se ha de hacer basándose en el contenido semántico de ambas frases. Las tres restantes son explícitas ya que el último término de una frase es idéntico al comienzo de la otra, por lo que deben resultar más fáciles de realizar. Los resultados se pueden ver en la Tabla IV. Los índices chi-cuadrado (g.l. 2) son significativos únicamente en HUR 1-2: $9.9(\mathrm{p}<.01)$ y SON 4-5: $9.9(\mathrm{p}<.01)$. La comparación entre pares de cursos me- 
diante la prueba de Fisher es significativa también únicamente en estas dos conexiones y exclusivamente en la comparación entre $50^{\circ}$ de E.G.B. y $3 .^{\circ}$ de B.U.P.F.P. Así, los resultados nos indican que hay diferencias significativas en dos de las conexiones locales difíciles, las oraciones 1-2 de «Huracanes» y las frases 4.5 de «Sonido», no encontrándose diferencias en las conexiones restantes. Como se puede ver en la tabla, en el caso de estas conexiones hay un incremento progresivo conforme avanza el grado académico de los chicos. En el caso de las conexiones más explícitas o fáciles ocurre que, o bien apenas hay diferencias, o bien las diferencias se dan entre $5 .^{\circ}$ nivel y el resto aunque sin llegar a ser significativas. Un caso especial lo constituye la conexión 3-4 de «Sonido» la cual es realizada únicamente por un $55 \%$ de los sujetos del curso más alto a pesar de que es una conexión considerada difícil. Si analizamos estas ordenaciones, vemos que hay algunos chicos mayores que realizan correctamente las otras dos conexiones poco explícitas, pero fallan en ésta porque conectan las oraciones 1-4 dentro de una secuencia general 2-1-4-5-6-3. Esta conexión 1-4, si bien es menos correcta que la 3-4, establece una conexión semántica entre «la vibración de los cuerpos» y «la excitación del tímpano» dentro de un esquema general coherente. Es decir, creemos que, a pesar de no realizar la conexión correcta, estos chicos mayores están empleando mecanismos de coherencia local guiados por una coherencia global del texto, basándose en su contenido semántico.

\section{TABLA IV}

Frecuencia y porcentaje de sujetos en la medida de "conexiones locales" $\left(N_{5.0}=12, N_{8.0}=12, N_{3.0}=11\right)$

\begin{tabular}{|c|c|c|c|c|c|c|c|c|c|c|c|c|}
\hline & \multicolumn{2}{|c|}{$\underset{1-2}{\text { HUR }}$} & \multicolumn{2}{|c|}{$\begin{array}{c}\text { SON } \\
3-4\end{array}$} & \multicolumn{2}{|c|}{$\begin{array}{c}\text { SON } \\
4.5\end{array}$} & \multicolumn{2}{|c|}{$\begin{array}{c}\text { HUR } \\
5.6\end{array}$} & \multicolumn{2}{|c|}{$\begin{array}{c}\text { SON } \\
1.3 \text { ó } 2-3\end{array}$} & \multicolumn{2}{|c|}{$\begin{array}{c}\text { SON } \\
5.6\end{array}$} \\
\hline & $\mathbf{0}$ & 1 & 0 & 1 & 0 & 1 & 0 & 1 & 0 & 1 & 0 & 1 \\
\hline $5^{\circ}$ & $\begin{array}{l}10 \\
73\end{array}$ & $\begin{array}{r}2 \\
17\end{array}$ & $\begin{array}{l}10 \\
83\end{array}$ & $\begin{array}{r}2 \\
17\end{array}$ & $\begin{array}{l}10 \\
83\end{array}$ & $\begin{array}{r}2 \\
17\end{array}$ & $\begin{array}{r}4 \\
33\end{array}$ & $\begin{array}{r}8 \\
67\end{array}$ & $\begin{array}{r}7 \\
58\end{array}$ & $\begin{array}{r}5 \\
42\end{array}$ & $\begin{array}{r}8 \\
67\end{array}$ & $\begin{array}{r}4 \\
33\end{array}$ \\
\hline $8 .^{\circ}$ & $\begin{array}{r}7 \\
58\end{array}$ & $\begin{array}{r}5 \\
42\end{array}$ & $\begin{array}{r}6 \\
50\end{array}$ & $\begin{array}{r}6 \\
50\end{array}$ & $\begin{array}{r}7 \\
58\end{array}$ & $\begin{array}{r}5 \\
42\end{array}$ & $\begin{array}{l}1 \\
8\end{array}$ & $\begin{array}{l}11 \\
92\end{array}$ & $\begin{array}{r}3 \\
25\end{array}$ & $\begin{array}{r}9 \\
75\end{array}$ & $\begin{array}{r}4 \\
33\end{array}$ & $\begin{array}{r}8 \\
67\end{array}$ \\
\hline $30^{\circ}$ & $\begin{array}{r}2 \\
17\end{array}$ & $\begin{array}{r}9 \\
83\end{array}$ & $\begin{array}{r}5 \\
45\end{array}$ & $\begin{array}{r}6 \\
55\end{array}$ & $\begin{array}{r}2 \\
17\end{array}$ & $\begin{array}{r}9 \\
83\end{array}$ & $\begin{array}{l}1 \\
9\end{array}$ & $\begin{array}{l}10 \\
91\end{array}$ & $\begin{array}{r}3 \\
27\end{array}$ & $\begin{array}{r}8 \\
73\end{array}$ & $\begin{array}{r}5 \\
45\end{array}$ & $\begin{array}{r}6 \\
55\end{array}$ \\
\hline
\end{tabular}

En cualquier caso, para confirmar nuestra clasificación de conexiones poco explícitas y muy explícitas, hallamos el coeficiente de contingencia entre las seis conexiones, encontrándose los resultados en la Tabla V. Tal como se puede ver, las conexiones poco explícitas tienen una alta correlación entre ellas, lo cual no ocurre en el caso de las conexiones explícitas. Es decir, parece que los chicos que efectúan las primeras tienen un determinado tipo de procesamiento semántico que está ausente en los otros sujetos.

Errores de ordenación. Estos errores nos pueden proporcionar datos adicionales sobre los mecanismos de coherencia empleados, así como sobre el dominio de claves lingüísticas generales. La Tabla VI presenta estos resultados. Debe hacerse notar que, en este caso, la calificación 1 indica «error», teniendo un valor negativo, a diferencia de los casos anteriores, mientras la calificación 0 indi- 
TABLA V

Coeficiente de contingencia entre las conexiones locales (Código: ${ }^{*}=p<.05 ;^{* *}=\mathrm{p}<.01$;

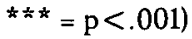

\begin{tabular}{lcccccc}
\hline & $\begin{array}{c}\text { HUR } \\
\mathbf{1 . 2}\end{array}$ & $\begin{array}{c}\text { SON } \\
\mathbf{3 . 4}\end{array}$ & $\begin{array}{c}\text { SON } \\
\mathbf{4 - 5}\end{array}$ & $\begin{array}{c}\text { HUR } \\
\mathbf{5 . 6}\end{array}$ & $\begin{array}{c}\text { SON } \\
\mathbf{5 . 6}\end{array}$ & $\begin{array}{c}\text { SON } \\
\mathbf{1 - 3 , 2 - 3}\end{array}$ \\
\hline HUR 1-2 & & $.5^{* * *}$. & $5^{* * *}$ & $.4^{*}$ & - & - \\
SON 3-4 & & & $.6 * * *$ & - & - & - \\
SON 4-5 & & & & - & - & - \\
HUR 5-6 & & & & & - & - \\
SON 5-6 & & & & & & - \\
SON 1-3, 2-3 & & & & & & \\
\hline
\end{tabular}

ca ausencia de error. Los índices chi-cuadrado (g.1. 2) son significativos en dos de los errores contemplados, ECOS 2-5 ó 5-2, 8.2 (p<.05), y «oración inicial inadecuada», $7.6(\mathrm{p}<.05)$. La comparación entre pares de cursos mediante la prueba de Fisher resulta igualmente significativa cuando comparamos $5 .^{\circ}$ y $8 .^{\circ}$ de E.G.B., y en la comparación de $5 .^{\circ}$ con $3 .^{\circ}$ de B.U.P.F.P. Es decir, en ambos casos las diferencias se producen entre el curso $5 .^{\circ}$ y el resto. Pensamos que el primer error de conexión se debe a repetición de términos, bien sea porque en ambos se habla de «medidas», o bien de «desequilibrios naturales». Es decir, hay términos que se repiten pero la conexión es claramente errónea si se atiende al significado de ambas frases. El otro error, colocación de oraciones inadecuadas en posición inicial, es revelador de las carencias sintácticas de muchos chicos de 5. de E.G.B., ya que esas oraciones no pueden ir en posición inicial porque en ellas se hace referencia a algún tipo de información previa. En el caso del error de colocación de las oraciones 4 ó 5 antes de 3 en el texto «Sonido» no hay diferencias significativas porque es un error poco cometido, incluso por los sujetos de $5 .^{\circ}(42 \%)$ y cometido por algunos de $8 .^{\circ}(25 \%)$ y $3 .^{\circ}(18 \%)$. Ello nos da pie para señalar un dato recurrente, como es que hay chicos con bajas destrezas de comprensión también en los cursos altos, lo cual hace que únicamente los errores cometidos por bastantes sujetos del curso más bajo posibilitan diferencias significativas con respecto a cursos mayores.

TABLA VI

Frecuencia y porcentaje de sujetos en la medida de uerrores de ordenación» $\left(N_{5,0}=12, N_{8 .}=12, N_{3 . o}=11\right)$

\begin{tabular}{|c|c|c|c|c|c|c|}
\hline & \multicolumn{2}{|c|}{$\begin{array}{l}\text { Ecosistemas } \\
2.5 \text { ó } 5.24\end{array}$} & \multicolumn{2}{|c|}{$\begin{array}{c}\text { Sonido } \\
4 \text { ó } 5 \text { antes de } 3\end{array}$} & \multicolumn{2}{|c|}{$\begin{array}{l}\text { Todos los textos } \\
\text { Orac. inic. inadecuad }\end{array}$} \\
\hline & 0 & 1 & 0 & 1 & 0 & 1 \\
\hline 5. E.G.B. & $\begin{array}{r}3 \\
25\end{array}$ & $\begin{array}{r}9 \\
75\end{array}$ & $\begin{array}{r}7 \\
58\end{array}$ & $\begin{array}{r}5 \\
42\end{array}$ & $\begin{array}{r}6 \\
50\end{array}$ & $\begin{array}{r}6 \\
50\end{array}$ \\
\hline $80^{\circ}$ E.G.B. & $\begin{array}{r}8 \\
67\end{array}$ & $\begin{array}{r}4 \\
33\end{array}$ & $\begin{array}{r}9 \\
75\end{array}$ & $\begin{array}{r}3 \\
25\end{array}$ & $\begin{array}{l}11 \\
92\end{array}$ & $\begin{array}{l}1 \\
8\end{array}$ \\
\hline $30^{\circ}$ B.U.P.-F.P. & $\begin{array}{r}9 \\
82\end{array}$ & $\begin{array}{r}2 \\
18\end{array}$ & $\begin{array}{r}9 \\
82\end{array}$ & $\begin{array}{r}2 \\
18\end{array}$ & $\begin{array}{l}10 \\
91\end{array}$ & $\begin{array}{l}1 \\
9\end{array}$ \\
\hline
\end{tabular}




\section{CONCLUSIONES}

En general, se puede decir que nuestras hipótesis han quedado confirmadas. Así, hay un incremento evolutivo patente en todas las medidas empleadas. Es decir, los chicos muestran una mejor ordenación de los texos conforme se incrementa el nivel académico, siendo significativas las diferencias entre los chicos de $5 .^{\circ}$ de E.G.B. y $3 .^{\circ}$ de B.U.P.F.P. en todos los textos, habiendo diferencias significativas entre los sujetos de $5 .^{\circ}$ y $8 .^{\circ}$ de E.G.B. en uno de los textos, y entre $8 .^{\circ}$ de E.G.B. y $30^{\circ}$ de B.U.P.-F.P. en otro de ellos. Resultados similares se producen cuando consideramos la variable supraordenación. Con respecto a las conexiones locales nuestra hipótesis también se confirma en líneas generales ya que dos de las tres conexiones poco explícitas arrojan diferencias entre el curso más alto y el más bajo, no siendo significativas las diferencias en el caso de las conexiones explícitas. Por lo que respecta a los errores de conexión las diferencias ocurren en dos de los tres errores, siendo significativas la diferencias entre el curso más bajo y cada uno de los dos restantes, lo que parece apuntar a que estos errores tan gruesos se superan tempranamente. Por lo que respecta a la comparación entre los tres textos, sobre lo cual no teníamos una hipótesis definida, encontramos que hay diferencias significativas en todos los textos si comparamos los chicos de 5..$^{\circ}$ de E.G.B. y $3 .^{\circ}$ de B.U.P.-F.P. Existen diferencias entre los sujetos de $8 .^{\circ}$ de E.G.B. y los de los cursos restantes, tanto el superior como el inferior, en algunos de los textos y en algunas de las medidas.

Todo lo anterior indica, a nuestro modo de ver, que gran parte de los sujetos de $3 .^{\circ}$ B.U.P.-F.P. (16 años) procesan los textos en base a aspectos semánticos y globales, es decir, establecen una coherencia global del texto que les sirve para ordenar y conectar las frases del texto a partir dicha globalidad, es decir, tienen un procesamiento acorde con el modelo de van Dijk y Kintsch (1983). Sin embargo, los sujetos de $5 .^{\circ}$ de E.G.B. manifiestan una notable ausencia de este procesamiento, estableciendo conexiones parciales entre frases basándose sobre todo en repetición de términos, pero sin llegar a un significado global integrador. Así, en ambos cursos se confirman los resultados expuestos en la introducción, tanto los de Scardamalia y Bereiter (1984), como los de otros autores que han encontrado deficiencias de procesamiento de texto en sujetos de 10-12 años. Estas deficiencias en procesos y estrategias de comprensión en lectores de los cursos medios de la escolaridad se han encontrado en tareas tales como la producción de textos (Scardamalia y Paris, 1985), la confección de resúmenes (Brown, Day y Jones, 1983; Winograd, 1984) o el recuerdo de textos (Meyer, 1984). En estos casos, los autores hablan de estrategias de «decir-cosas-quesabemos» (knowledge-telling strategies en la producción de textos), "copiarborrar» (copy-delete en el resumen), y «estrategia de listado» (default list strategy para el recuerdo). Todas estas estrategias serían equivalentes a la calificada por Scardamalia y Bereiter (1984) como tópico-más-detalles la cual mencionamos en la introducción.

Por otra parte, los sujetos de $8 .^{\circ}$ parecen encontrarse, en general, en una fase de transición en la cual hay elementos de coherencia global que no llegan, en muchos casos, a producir una integración total de las frases, quizás debido, entre otros aspectos, a unas carencias de capacidades sintácticas y esquemáticas, con respecto a los procesos de organización de los textos. Naturalmente, en todos los cursos hay diferencias individuales por lo que hay chicos que podrían encuadrarse dentro de un nivel de procesamiento superior o inferior al 
propio de su edad, tal como lo hemos descrito aquí. No obstante, queremos analizar con más precisión algunos de los resultados.

En primer lugar, como hicimos notar en el apartado de resultados, no hay ningún chico de $8 .^{\circ}$ que ordene correctamente el texto «Huracanes». Sin embargo, cuatro de los chicos dan la ordenación 1-2-5-6-3.4, lo cual ocurre también en dos de los chicos mayores. Esta ordenación tiene el esquema que se puede ver el la figura 3. Este esquema es idéntico a la ordenación adecuada, a excepción de que los efectos están cambiados de orden. Ello es así porque la oración 5 comienza diciendo: «los huracanes también suelen ...» y en la frase 6 se lee» «estas lluvias, lo mismo que las olas altas, pueden ...». Así, las expresiones en cursiva nos están indicando que se ha debido hablar de un efecto anterior, y de que en ese efecto se ha debido nombrar a las «olas». En definitiva, creemos que los chicos que hacen la ordenación que estamos comentando procesan semánticamente, pero tienen unas carencias de tipo sintáctico que les hacen cometer algunos errores.

FiguRA 3

1. Huracanes: definición y características

2. Efectos de los buracanes:

2.1: Ocasionan lluvias, que pueden causar inundaciones

2.2: Ocasionan olas en el océano, que pueden bundir los barcos

Esquema de ordenación del texto «Huracanes» según algunos chicos mayores

Estas carencias se pueden apreciar también en el bajo número de chicos de $8 .^{\circ}$ que tienen una buena supraordenación en «Sonido». La mayoría de los que no colocan la oración inicial en posición correcta invierten la ordenación, colocando 2-1 en lugar de 1-2, lo cual también ocurre con algunos chicos mayores, pero con ninguno de los de $5 .^{\circ}$. Si se analizan las dos oraciones, se puede apreciar que la 2 ( Si haces sonar una campana de cristal e inmediatamente acercas débilmente la uña al borde de la copa, sentirás la vibración») está mejor colocada después de la 1 («El sonido es producido por la vibración de los cuerpos») porque es un ejemplo de ésta, y además, porque hay un artículo determinado que acompaña al término «vibración» que está indicando que dicho término se ha debido nombrar anteriormente. Así, se confirma una falta de dominio de las estructuras esquemáticas de la prosa (principio-ejemplo), y de algunos conocimientos sintácticos (función del artículo determinado en la prosa expositiva) (Kieras, 1985), en estos chicos de $8 .^{\circ}$ que estarían en una fase de transición. Obviamente, estas carencias son mucho más patentes en los chicos de $50^{\circ}$ tal como se puede apreciar por las bajas puntuaciones de supraordenación y conexiones locales, sobre todo en aquellas poco explícitas, y el considerable número de errores de ordenación. Estas carencias en supraordenación coinciden no sólo con el estudio de Garner y Gillingham (1987), sino también otros varios que constatan la dificultad de los escolares de grados intermedios para captar la ideas principal de textos expositivos (Williams, Taylor y Ganger, 1981; Hare y otros, 1989).

Una segunda cuestión que queríamos comentar se refiere a las diferencias entre los textos. Ciertamente la variable texto no introduce diferencias significativas, lo cual parece indicar que existe un determinado tipo de procesamiento que es independiente del pasaje que se lea. Ahora bien, hay textos como el de «Huracanes» en el que las puntuaciones de supraordenación son considerable- 
mente más altas que en el resto, dicho esto en general pero especialmente para los chicos de $5 .^{\circ}$. Creemos que la explicación está en que la palabra tópica del texto («los huracanes») está muy repetida a lo largo del texto, lo cual no ocurre en los otros pasajes. Así, el tema es fácilmente detectable, siendo la primera oración la más genérica («un buracán es ...») ya que se da la definición del término. Así, tanto los elementos sintácticos de las frases (término «huracanes» en posición de sujeto), como los conocimientos esquemáticos de la prosa (comenzar un texto por la definición del objeto que se describe) o la repetición de términos, pueden facilitar la comprensión, lo cual es coherente tanto con los modelos macroestructurales (van Dijk, Kintsch, 1983) como con las explicaciones basadas en modelos mentales (Sanford y Garrod, 1982).

Los resultados obtenidos creemos que pueden tener interés para dos cuestiones aplicadas. Una es el diagnóstico de habilidades de comprensión. La tarea de ordenación de textos puede proporcionar gran número de datos al respecto, tal como hemos mostrado. La segunda aplicación se deriva de su contribución para establecer una secuencia evolutiva de procesos de comprensión que ayude a elaborar programas de intervención que tengan en cuenta el nivel de desarrollo cognitivo de los sujetos. Ciertamente se necesita más investigación al respecto, pero existen trabajos como los que hemos comentado y otros realizados desde un marco más estructurado de desarrollo cognitivo (Case y McKeough, 1989) que están contribuyendo a dibujar un panorama cada vez más claro sobre los procesos evolutivos en la comprensión de la prosa.

Además de estas repercusiones, pensamos que hay cuestiones abiertas que deberían ser abordadas en estudios posteriores. Una de ellas es la pregunta de hasta qué punto los resultados de nuestro estudio reflejan datos de procesos generales de comprensión que puedan ocurrir en otras tareas típicas de comprensión y de recuerdo. La segunda cuestión abierta está estrechamente relacionada con la anterior y se refiere a la posibilidad de que un entrenamiento en estrategias generales de comprensión se generalice a una tarea como la de ordenación de frases. Si así fuera, podríamos estar más seguros de que estamos midiendo procesos generales de comprensión y no procesos específicos de una tarea concreta. Esta línea de investigación de procesos apoyándose en estudios instruccionales constituye una de las vías más prometedoras en la investigación sobre el aprendizaje de procesos mentales complejos (Glaser y Bassok, 1989). La tercera cuestión se refiere a la relación entre medidas metacognitivas, como las tomadas en el estudio de Scardamalia y Bereiter mediante un procedimiento de pensar-en-voz-alta, y medidas de ejecución como la nuestra. Se debería comprobar el grado de coincidencia entre ambos tipos de medidas, ya que los problemas de medición son uno de los retos más importantes de la psicología cognitiva en general y de la comprensión lectora en particular. Por último, habría que investigar cómo el conocimiento previo del contenido del texto afecta a las estrategias de comprensión en una tarea como la que hemos utilizado. Esta variable es de suma importancia en los procesos de comprensión en general y deberá ser relevante también en este caso.

\section{Notas}

(1) Si agrupamos los datos de los sujetos de los dos cursos superiores con el fin de cumplir más estrictamente con las restricciones de utilización de la prueba chi-cuadrado (ver Siegel, 1978, p. 209), los resultados son idénticos, por lo que hemos preferido, tanto en este caso como en los siguientes, proporcionar las puntuaciones por curso. 


\title{
Referencias
}

BREwer, W. J. (1980). Literary theory, rhetoric and stylistics: implications for Psychology. En R.J. Spiro, B.C. Bruce, W.F. Brewer (Eds.), Theoretical issues in reading comprehension. Hillsdale, N.J.: Lawrence Erlbaum Associates.

Brown, A. L.; DAY.J. D., Jones. R. S. (1983). The development of plans for summarizing texts. Child Development, 54, 968-976.

CASE, R., y MCKEOUGH (1989). Schooling and the development of central conceptual structures: an example from the domain of children's narratives. International Joumal of Educational Research, $835-855$

ENGLert, C. S. y Hiebert, E. H. (1984). Children's developing awareness of text structure in expository materials. Joumal of Educational Psychology, 76, 1, 65-74.

ENGLERT, C. S., y THOMAS, C. Ch. (1987). Sensitivity to text structure in reading and writing: a comparaison between learning disabled and non-learning disabled students. Leaming Disability Quarterly, Spring, 93-105.

Garner, R. (1987). Metacognition and reading comprebension. Norwood, N.J., Ablex Publishing Corporation.

Garner, R., y Gillingham. M. G. (1987). Students' knowledge of text structure. Joumal of Reading Behavior, XIX, 3, 247-259.

Glaser. R., y BASSOK M. (1989). Learning theory and the study of instruction. Annal Review of Psychology, 40, 631-666.

Graesser, A. C., y Goodman, S. M. (1985). Implicit knowledge and the representation of expository text. En B.K. Britton y J.B. Black (Eds.), Understandig expository text. Hillsdale, N.J., Lawrence Erlbaum Associates.

Hare. V. C.; Rabinowitz, M., y SchniEble, K. M. (1989). Text effects on main idea comprehension. Reading Research Quarterly, XXIV, 1, 72-88.

Kieras. D. E. (1985). Thematic processes in the comprehension of technical prose. En B.K. Britton y J.B. Black (Eds.), Understandig expository text. Hillsdale, N.J., Lawrence Erlbaum Associates.

MAYER. R. E. (1985). Structural analysis of science prose: can we increase problem-solving performance?. En B.K. Britton y J.B. Black (Eds.), Understandig expository text. Hillsdale, N.J., Lawrence Erlbaum Associates.

MEYeR, B. J. F. (1984). Text dimensions and cognitive processing. En H. Mandl, N.L. Stein y T. Trabasso (Eds.), Leaming and comprebension of text. Hillsdale, N.J., Lawrence Erlbaum Associates.

MEYER, B. J. F. (1985). Prose analysis: purposes, procedures and problems. En B.K. Britton y J.B. Black (Eds.), Understandig expository text. Hillsdale, N.J., Lawrence Erlbaum Associates.

Richigess, D. J.; McGee, L. M.; Lomax, R. G., y Sheard, C. (1987). Awareness of four text structures: effects on recall of expository text. Reading Research Quarterly, XXII, 2, 177-196.

SANFORd, A. J., y Garrod, S. C. (1982). Undertanding written Language. New York, Wiley \& Sons.

Scardamalia M., y Bereiter. C. (1984). Development of strategies in text processing. En H. Mandl, N.L. Stein y T. Trabasso (Eds.), Learning and comprehension of text. Hillsdale, N.J., Lawrence Erlbaum Associates.

Scardamalia, M., y Paris, P. (1985). The function of explicit discourse knowledge in the development of text representations and composing strategies. Cognition and instruction, 2(1), 1-39.

Siegel, S. (1978). Estadistica no paramétrica. Mexico, Trillas.

SprRo, R. J., y TAYLOR, B. M. (1987). On investigating children's transition from narrative to expository discourse: the multidimensional nature of psychological text classification. En R.J. Tierney, P.L. Anders, J.N. Mitchell (Eds.). Understanding readers' understanding. Hillsdale, N.J., Lawrence Erlbaum Associates.

Van Dijk. T. A., y KinTsch. W. (1983). Strategies of discourse comprehension. New York, Academic Press.

Willams. J. P.; TAylor, M. B., y Ganger, S. (1981). Text variation at the level of the individual sentence and the comprehension of simple expository paragraphs. Joumal of Educational Psycho$\log y, 73,851-865$.

WINOGRAD, P. N. (1984). Strategic difficulties in summarizing texts. Reading Research Quarterly, 19. 404-425. (Traducido en Infancia y Aprendizaje, 31-32, 67-87).

\section{Anexo}

\section{CRITERIOS EMPLEADOS PARA LA PUNTUACION DE LA ORDENACION DE TARJETAS EN CADA UNO DE LOS TEXTOS.}

\author{
TEXTO 1. ECOSISTEMAS
}

- 4 puntos: orden correcto 
- 3 puntos: se colocan las tarjetas 1-2-3 en ese orden y en posición inicial (en ellas se define lo que se entiende por «equilibrio de los ecosistemas», se enuncian los dos tipos de medidas que hay que tomar, y se desarrolla la primera de ellas)

- 2 puntos: $O$ bien se colocan las tres tarjetas iniciales en orden (1-3-2-) y el resto de las tarjetas está en posición correcta, o bien se mantiene la conexión que se considera fundamental (1-2) (se define lo que se entiende por «equilibrio de los ecosistemas», se enuncian los dos tipos de medidas que hay que tomar), aunque no esté colocada al comienzo

- 1 punto: coloca la tarjeta 1 en la posición correcta

- 0 puntos: cualquier otra ordenación

\section{TEXTO 2. HURACANES}

- 4 puntos: orden correcto

- 3 puntos:

a) tiene la tarjeta inicial en posición correcta, y

b) los cambios no afectan a tres conexiones semánticas que son básicas para comprender el texto $(1-2 ; 3-4$ y $5-6)$

- 2 puntos:

a) tiene la tarjeta inicial en posición correcta, y

b) se conservan al menos dos de las tres conexiones referidas anteriormente

- 1 punto:

O bien

a) tiene la tarjeta inicial en posición correcta, y

b) tienen alguna de las conexiones aludidas anteriormente

$\mathrm{O}$ bien, conservan al menos dos de las tres conexiones referidas anteriormente, aunque no tengan la tarjeta inicial en posición correcta

- 0 puntos: cualquier otra ordenación

\section{TEXTO 3. SONIDO}

- 4 puntos: orden correcto

- 3 puntos:

O bien:

a) tiene la tarjeta inicial en posición correcta, o bien la tiene en segundo lugar pero precedida de la $n .^{\circ} 2$ (es decir, comienza con 2-1), y

b) las colocaciones erróneas no son las dos que se consideran esenciales para poder comprender el proceso del sonido $\left(1 .^{\circ}\right.$ la tarjeta 3 precedida de la 4 ó $5 ; 2 .^{\circ}$ la tarjeta 4 precedida de la 5)

O bien:

- coloca la tarjeta 1 en posición final y todas las demás están correctas

- 2 puntos:

a) tiene la tarjeta inicial en posición correcta, o bien la tiene en segundo lugar pero precedida de la n. ${ }^{\circ} 2$ (es decir, comienza con 2-1), y

b) hay sólo alguna de las dos colocaciones incorrectas mencionadas

- 1 punto: tiene la tarjeta inicial en posición correcta, o bien la tiene en segundo lugar pero precedida de la n. 2 (es decir, comienza con 2-1)

- 0 puntos: cualquier otra ordenación. 


\section{Extended Summary}

According to van Dijk and Kintsch's model (1983) fluent readers employ procedures of local and global coherence in text processing. The result of these procedures is the construction of a situational model in which textual information is intergrated in the reader's knowledge structures. Sentence arragement tasks in which the subject has to construct a coherent text permits to investigate these procedures and the resulting mental model.

Scardamalia and Bereiter (1984) conducted a study in which this task was employed using a thinking-aloud methodology. They found there were differences between 12 and 16 year old students (6th and 10th grade respectively). In the case of the older students, the protocol analysis showed that they used mature strategies which, according to van Dijk and Kintsch's model, involved grasping the text's global meaning. In contrast, younger students exhibited immature strategies, suggesting that they employ a text processing model that proceeds element by element, and relying to a large extent on superficial text characteristics that can be extracted without carrying out a deep analysis.

The above study poses several issues that were considered in our own research. First, the great age difference between both subject groups makes it difficult to analyze the stage of transition from immature to mature sentence arrangement strategies. Secondly, provided that expository prose is different from narrative prose in several schematic and surface characteristics, it is possible that at least some of the results would depend on the type of prose that is used. This suggests the convenience of studying more carefully sentence arrangement processes through expository texts with different textual structures. Finally, the use of metacognitive measures should be complemented with other measures of performance because the first have some validity problems, -at least when they are employed with middle-grade school children. In fact, in a study with 5th and 7th grade school children, Garner and Gillingham (1987) found little correspondence betweem both types of measures when they evaluated subjects' knowledge of some structural text properties through a task similar to sentence arrangement.

Thus, the present study attempted to partially replicate Scardamalia and Bereiter's work. The difference lies in that in our work some changes, congruent with the above mentioned issues, were introduced. The first change was to include three different age groups (i. e., 10,13 and 16 years old students) in order to capture subjets' transition stages in processing strategies. Secondly, only expository texts with different textual structures were used. Finally, the study measured the result of sentence arrangement rather than subjects' verbal reports. It was predicted that students would show a gradual improvement in correct sentence arrangement as well as in superordination and local cohesion, while increasing their academic level. There was no previous hypothesis about the effect the textual structures would have on the sentence arrangement task.

Thirty-five students distributed in three groups, 12 students 10 years old, 12 students 13 years old, and 11 students 16 years old, participated in the study. All subjects read three expository texts (length: 78-132 words), each divided into six different sentences that had to be arranged to construct a coherent text. Each sentence was presented on a separate piece of paper. The texts used were adaptations from school textbooks and a school encyclopedia. They were then 
independently arranged by three Philology University lecturers who confirmed the correct sentence order. Subjects were examined by specially trained undergraduated students from a Teacher's Training College. Four types of measures were taken for each text. First, an index of general sentence arrangement was taken, consisting on an ordinal measure with a value ranging from 0 to 4 depending on local and global coherence criterion. Second, sentence superordination was measured; one point was given if students put the initial sentence in the right place and 0 if they did not. Third, six local connections between pairs of sentences were evaluated; three referred to explicit connections, and the other three to the global meaning of sentence pairs without explicit referents. Fourth, the last measure looked at significant errors in sentence arrangement.

Results generally confirmed our hyphotheses. In general sentence arrangement older students obtained higher scores than the younger ones. For each text, significant differences were found between 16 year old subjects and 10 year old ones. On one text, 13 year old children obtained significantly higher scores than the 10 year old group, and on a different text the 13 year old group obtained significantly lower scores than 16 year old students. These children seem to be in a transitional stage which involves the use of global coherence procedures but they lack certain syntactic and schematic skills. A similar trend can be observed in relation to supraordination. In two out of the three less explicit local coherence ties similar results were obtained; but in this case differences between the intermediate age group and the other two groups, lower and higher, were not significant. In terms of explicit ties, although the youngest group scored lower than the rest, no significant differences among age groups were found. However, an opposite developmental trend was found in errors of sentence arrangement. The lowest age group made more mistakes than the two older groups, and two out of the three scores were significant. So it seems that we are measuring strategic skills that are independent from the kind of text employed, though these textual variations particularly affect children who are in a transitional stage.

We think that these results could have educational repercussions on assessment and intervention on reading comprehension difficulties. We also believe that additional research studies are needed to analyze to what extent the skills measured in the study are related to other general comprehension abilities. 